\title{
Pediatric PET/CT Imaging: Tips and Techniques*
}

\author{
Susan McQuattie \\ Division of Nuclear Medicine, Department of Diagnostic Imaging, Hospital for Sick Children, Toronto, Ontario, Canada
}

\begin{abstract}
This article was written to give health-care providers working in the field of nuclear medicine some background information on pediatric PET/CT. Specifically, it provides information regarding patient preparation and acquisition techniques necessary to obtain high-quality pediatric PET/CT images. It is targeted primarily at nuclear medicine technologists and CT technologists but may be beneficial to physicians performing PET/CT scans as well. The learning objectives for this article are to help the reader understand the practical aspects involved in pediatric PET/CT, to provide helpful tips and techniques that can be applied to pediatric nuclear medicine, and to help the reader understand and explore the various studies being done with ${ }^{18} \mathrm{~F}-\mathrm{FDG}$ in children.
\end{abstract}

Key Words: pediatric; PET/CT; technique

J Nucl Med Technol 2008; 36:171-178

DOI: 10.2967/jnmt.108.051995

$\mathbf{T}$ he use of ${ }^{18} \mathrm{~F}-\mathrm{FDG}$ in PET/CT is well documented as a valuable tool in the staging and diagnosis of disease for many oncology patients. Although considered routine in adults, PET/CT in children has been somewhat limited. The recent acquisition of a Phillips Gemini GXL PET/CT scanner at The Hospital for Sick Children in Toronto, Ontario, Canada, has provided both clinicians and families with access to a service that previously required traveling great distances at personal expense. The purpose of this article is to familiarize technologists and clinicians with some of the practical aspects involved in successful pediatric PET/CT and explore some of the other PET/CT applications currently being used on children.

\section{PATIENT PREPARATION}

The key to a successful PET scan begins with appropriate patient preparation, and pediatric imaging is no exception to this rule. When possible, information about the procedure should be given beforehand through information sheets sent to the family or through a phone call with preparation instructions. Parents can be confused by the complexity of $\mathrm{PET} / \mathrm{CT}$, and ample time must be allotted to explain the

\footnotetext{
Received Feb. 25, 2008; revision accepted Aug. 4, 2008.

For correspondence or reprints contact: Susan McQuattie, Hospital for

Sick Children, 555 University Ave., Toronto, Ontario, Canada M5G 1X8.

E-mail: susan.mcquattie@sickkids.ca

*NOTE: FOR CE CREDIT, YOU CAN ACCESS THIS ACTIVITY THROUGH THE SNM WEB SITE (http://www.snm.org/ce_online) THROUGH DECEMBER 2010.

COPYRIGHT (C) 2008 by the Society of Nuclear Medicine, Inc.
}

study and allow the parents and patient an opportunity to ask questions (1). Patients are asked to fast for at least $4 \mathrm{~h}$ before injection to reduce the glucose level and to lower circulating insulin levels, thus optimizing the target-to-background ratio. Glucose levels are checked for each patient before ${ }^{8} \mathrm{~F}-\mathrm{FDG}$ injection and must be less than $11 \mathrm{mmol} / \mathrm{L}$, or $150 \mathrm{mg} / \mathrm{dL}$ (2), in order to proceed. Patients are permitted a small amount of water if they must take oral medications before arriving for the scan. If the patient is diabetic, the issue of fasting is discussed with the referring physician and with the nuclear medicine physician and may require consultation with a diabetic-control specialist $(2,3)$. On arrival in the nuclear medicine department, the patient's height and weight are obtained, and the patient and parent are brought into a private room where they will remain during the uptake phase of the scan. The procedure is explained to both the patient and the parent, and any questions or concerns can be addressed as required. A concise and thorough patient history should also be taken at this time and must include information on the patient's last chemotherapy or radiation therapy session, any medications the patient is currently taking, and the date of the patient's last menstrual cycle if applicable. Any indwelling lines such as a peripherally inserted central catheter or central venous line, and their location on the body, should also be noted.

\section{COMMUNICATION}

Communication has always been an essential tool for the nuclear medicine technologist and becomes even more important when dealing with pediatric patients. A good technologist develops the skills necessary to talk not only to the parents but also to the patients at an appropriate level of understanding. Communication can be challenging, as parents are often distracted, having to watch their child while listening to explanations of procedures that may be new and foreign to them. It is important to be aware of these distractions and recognize signs that the information is not being received as effectively and thoroughly as possible. Some helpful suggestions include having one parent deal with the child while the other listens to the explanation, or perhaps having a second technologist distract the child through play and visual stimulation if both parents are not available. It is essential that the parent or patient be given time to ask questions or express concerns at any point, particularly when one is dealing with frightened or anxious children, who may be less cooperative if they do not 
understand what is happening to them. Often a simple explanation of what they will be experiencing and what you will be doing to them can alleviate much anxiety. It is also important to keep the patient's age in mind when conveying information. A good pediatric imaging technologist should know what to expect from the child's age group yet be mindful that some children may be at different stages of maturity, psychosocial development, and cognitive capacity (1). There are many guides available that outline the various stages of child development (4). After assessing the patient by speaking with the parent or child, the technologist can effectively adjust techniques to suit each situation. Good communication skills are not limited to the exchange between a technologist and the patient or parent. In some institutions, the PET/CT scanner is away from the nuclear medicine department and may be used as a dedicated CT scanner when no PET/CT patients are scheduled. In this case, it becomes imperative that a good line of communication exist between the 2 modalities, from scheduling clerks to technologists to physicians. When a request is received, scheduling time must be coordinated with the CT department to ensure the availability of the scanner and of technologists trained in PET/CT. This communication must continue throughout the entire process and becomes crucial on the day of the examination. Once the patient has arrived and the procedure has been explained, a quick phone call to the scanning room to ensure that the requested time is a suitable one for injecting the patient can alleviate many frustrations and delays. This verification phone call allows the CT technologists the opportunity to schedule other patients as required that day and ensures that the scanner can be used to its fullest potential. Any unforeseen delays can then be communicated to the parents and patients, thus avoiding unrest and uneasiness for all.

\section{SEDATION AND PATIENT DISTRACTION TECHNIQUES}

The issue of cooperation in children presents many challenges to nuclear medicine technologists, and children undergoing a PET/CT scan are no exception. For many children, undergoing a PET/CT examination may not be their first experience in a nuclear medicine or CT department, and a sense of familiarity with the equipment and staff in the department may already exist. As previously mentioned, good communication is essential to obtaining a successful PET/CT scan. A child who has undergone previous CT or nuclear medicine procedures may feel more at ease about undergoing PET/CT once the similarities between the procedures have been explained. If time permits, it may be helpful to show the child the scanner before injection so that the child can better understand what will be happening during the acquisition and thus be less anxious about the procedure. It can also be helpful to allow the child to bring a favorite toy or stuffed animal into the scanning room if possible. This toy can be placed above the head of the child or held in the child's hands above the head out of the field of view. Allowing a parent to remain in the room during the scan can also give the child a sense of security and may help an otherwise uncooperative child to successfully complete the scan without the need for sedation. Parents must be clearly instructed on what they can do to help, as well as what is required of the child in order for the study to be performed. It is possible that the parents may be familiar with the CT aspect of the scan but not with the PET portion and thus may not realize the length of time required to complete the PET scan. Again, clear and concise communication about what will be happening as the scanning bed moves into the PET scanner is essential. It is not uncommon for both parents and patients to be unaware of imaging taking place, as they will not see or hear anything after the bed moves to each new position.

With many nuclear medicine procedures, the use of a DVD or videocassette player can be vital in obtaining patient cooperation throughout imaging. Patients can often have their head turned to the side to see the television screen for much of the study. This can be a valuable distraction technique and for many younger patients is enough to obtain good-quality images. Unfortunately, a PET/ CT scanner does not routinely allow the use of such devices, as the patient is required to lie with the head straight and arms up for the entire acquisition. In such cases, it may be useful to have a radio or $\mathrm{CD}$ player available and ask the parents and child what music they would like to listen to during the test. Alternatively, the parent or technologist can offer to read from a book the child may currently be enjoying or sing favorite and familiar songs to the child. This type of distraction helps the children to feel safe and reassures them that they are not alone in the room. Older children and teenagers will often not require any distraction techniques and will be able to lie still and quietly for the duration of the scan. Many find the procedure quite comfortable and will fall asleep during the study. In these instances, the parents may choose to wait outside the scanning room until imaging is complete.

The need for sedation in children must be assessed on an individual basis. Younger children may be able to cooperate for a CT scan; however, the lengthier time needed for a PET scan may require the child to be sedated. If sedation is required, in most cases it will be considered only for the imaging portion of the study. Once the need to sedate has been established, the technologist must involve other professionals in the administration of the sedative, continuous monitoring, and recovery after the procedure. Guidelines and methods will vary according to different departments and institutions, and the technologist must confirm that the required preparation instructions for sedation have been followed before the child is injected for the scan (1). Timing of the PET/CT scan may need to be altered somewhat if the patient is to be scanned under sedation or a general anesthetic, as administration of the sedative can sometimes be time consuming. It may become necessary to 
bring the patient into the scanning room before the uptake phase has been completed. Often, allowing the patient to remain in the uptake room for $45 \mathrm{~min}$ before initiating sedation is sufficient both from a technical and from a practical point of view. This timing can be discussed with the appropriate professionals before the start of the study to ensure a smooth progression throughout the study. All personnel involved with the patient should be familiar with the patient positioning and duration that will be required for the PET/CT scan so that medical equipment and patient intravenous lines can travel safely with the patient through the scanner and patient safety can be maintained. It may also be helpful to tell nurses and physicians involved with sedating the PET/CT patient that although they will not be able to be in the room with the patient during the CT acquisition, once the PET acquisition has commenced they will be allowed to enter the room should it be necessary. Having appropriate personnel in the room can become important when patient monitors are not easily viewed from the control room or if adjustments are needed to maintain an appropriate level of sedation during the scan. Once scanning is complete, the images should be reviewed before the patient is transferred off the scanning bed to ensure that no further imaging is required. This review will normally require only 5-10 min and can lessen the likelihood of having to repeat a scan once the sedative has begun to wear off. With appropriate preparation and careful planning, one can use sedation for PET/CT scans with minimal disruption to workflow and can allow for superior image acquisition when patient cooperation cannot be achieved by any other means.

\section{${ }^{18}$ F-FDG ADMINISTRATION AND UPTAKE}

The recommended dose of ${ }^{18} \mathrm{~F}$-FDG for pediatric use is in the range of $5-10 \mathrm{MBq} / \mathrm{kg}$, or $0.15-0.30 \mathrm{mCi} / \mathrm{kg}$, with a minimum dose of $37 \mathrm{MBq}$ (1 mCi) (5). The Hospital for Sick Children uses a maximum of $370 \mathrm{MBq}(10 \mathrm{mCi})$ for wholebody ${ }^{18}$ F-FDG imaging. For infants, the dose is routinely discussed with the nuclear medicine physician and may be adjusted according to the procedure the patient will be undergoing and any underlying medical issues. When one is injecting patients for PET scans, it is imperative that a preand postdose measurement be done and recorded with the patient information. These values and times are used in the calculation of the standardized uptake value, and inaccuracies can lead to incorrect uptake values. If unusual standardized uptake values are noted for a patient, they may be the result of an inaccurate entry into the computer at the time of acquisition. The exact time that the pre- and postdose measurements are obtained is noted, along with the exact time of injection. Establishing a patient worksheet as in Figure 1 can help to ensure all data are recorded correctly and will help eliminate inaccuracies as a source of incorrect standardized uptake value calculations.

As with any pediatric nuclear medicine procedure, intravenous access can be problematic depending on patient cooperation and hydration status. Although many pediatric patients will have an established central venous line or a subcutaneous port, injection through these devices is not always possible. Because some of these patients will be undergoing diagnostic $\mathrm{CT}$ with intravenous contrast material as part of the procedure, it is important to check with the staff in the CT department as to the suitability of the line. In many instances, the nuclear medicine technologist will start a peripheral intravenous line before injection of the ${ }^{18} \mathrm{~F}-\mathrm{FDG}$. This eliminates the possibility of dose extravasation, which could lead to lymph node uptake and reconstruction artifacts if the hot injection site is in the field of view (1). In addition, establishing an intravenous line before injection allows the patient time to recover, as the experience can often be painful and stressful (particularly if access has been difficult and multiple attempts have been made), resulting in increased muscle uptake. If a central venous line, peripherally inserted central catheter, or subcutaneous port is to be used, a good flush must be done to ensure minimal residual activity within the line. At the time of injection, patients are given a comfortable reclining chair to sit in and are encouraged to remain quiet and calm. It is also essential to ensure that the patients are kept warm to reduce uptake in brown fat. Most often, a warm blanket or flannel sheet wrapped around the shoulders and body will suffice.

Perhaps one of the most challenging aspects of pediatric PET/CT involves the uptake phase. Although it is important for the patients to remain comfortably in a warm room for 45-60 min, with minimal activity and talking, this requirement can present a challenge for younger patients. One parent is usually allowed to remain in the room with the patient and must understand the importance of minimizing activity and of helping, when possible, to achieve this goal. When possible (and if deemed appropriate by the parents), a television can be brought into the room for the child to watch, to aid in minimizing movement. Other distractions and relaxation techniques include listening to soft music or having a parent read to the child. Using portable game devices and having the patient hold a book to read are discouraged as these can lead to increased muscle use and therefore increased uptake in those muscles. The uptake phase for most studies using ${ }^{18} \mathrm{~F}-\mathrm{FDG}$ ranges from 30 to $60 \mathrm{~min}$, depending on the procedure being performed. Some studies such as brain and cardiac imaging require less uptake time. Muscle relaxants and other pharmaceuticals can also be used to calm an anxious or claustrophobic child. However, in most instances these will not be required. Parents of patients who are known to be anxious during procedures will often inquire at the time of booking what options exist so that suitable arrangements can be made ahead of time. On occasion, you may encounter a patient in whom involuntary muscle movement cannot be avoided and increased uptake may be seen on the PET/CT scan. Such involuntary movement should be noted on the patient's requisition or worksheet and communicated to the physician so that any increased uptake in these areas can be taken into consideration when a report is made. An 
PET/CT Technical Data Sheet

\begin{tabular}{|c|c|}
\hline \multirow[t]{2}{*}{ Date of Exam: } & Time In: \\
\hline & Time Out: \\
\hline \multicolumn{2}{|l|}{ Patient Name } \\
\hline \multicolumn{2}{|l|}{ MRN } \\
\hline \multicolumn{2}{|l|}{ Height } \\
\hline \multicolumn{2}{|l|}{ Weight } \\
\hline \multicolumn{2}{|l|}{ Last Menstrual Period (if applicable) } \\
\hline Glucose Level & $\mathrm{mmol}_{\mathrm{L}} \mathrm{L}$ at $\quad \ldots \mathrm{hrs}$ \\
\hline Pre Dose & $\mathrm{mCi}$ at \\
\hline Post Dose & $\mathrm{mCi}$ at \\
\hline${ }^{18}$ F-FDG dose & $\mathrm{mCi}_{\ldots}$ at \\
\hline \multicolumn{2}{|l|}{ FDG Lot \# } \\
\hline \multicolumn{2}{|l|}{ IV Injection Site } \\
\hline \multicolumn{2}{|l|}{ Start Time of Image Acquisition } \\
\hline \multicolumn{2}{|l|}{ End Time of Image Acquisition } \\
\hline Was Diagnostic СТ Performed? & $\begin{array}{l}\text { Y or } \mathbf{N} \text { (circle one) } \\
\text { If Yes, Was Intravenous Contrast } \\
\text { Given? (circle one) Y or N }\end{array}$ \\
\hline If Intravenous Contrast Was Given for CT $\rightarrow$ & $\begin{array}{l}\text { Type: Visipaque or Omnipaque (circle one) } \\
\text { Injection Method: Power or Hand (circle one) } \\
\text { Volume: } \quad \mathrm{mL}\end{array}$ \\
\hline СT Technique & $\mathrm{mAs}=$ \\
\hline Sedation Type & $\begin{aligned} \text { (check one:) } & \square \text { No Sedation } \\
& \square \text { Conscious Sedation } \\
& \square \text { General Anesthetic }\end{aligned}$ \\
\hline Bladder Catheter? & $\mathbf{Y}$ or $\mathbf{N}$ (circle one) \\
\hline
\end{tabular}

FIGURE 1. Example patient worksheet to help ensure that all data are recorded correctly and to help eliminate inaccuracies in standardized uptake value calculations. radiation exposure to the technologist (1). Parents and patients should be made aware of this device if one is in use and should also be instructed on what to do if urgent care is required. The use of a call button similar to ones found in most patient hospital rooms is an effective method for maintaining patient safety, particularly if no monitoring camera is available.

\section{ACQUISITION}

When the ${ }^{18} \mathrm{~F}-\mathrm{FDG}$ uptake phase is complete, the child is asked to void before entering the scanning room to eliminate bladder activity from the images and ensure the child's comfort during the scan (1). Bladder catheterization is not routinely used at The Hospital for Sick Children for PET/CT scans but has been used for patients who are imaged under general anesthetic. In that instance, the catheter can be inserted after administration of the anesthetic and removed once the bladder has been completely drained. It is important that parents be made aware of the possibility that their child may be catheterized while under anesthesia and will not experience any discomfort from the procedure.
FIGURE 2. Transaxial projection of head revealing extraocular muscle uptake (arrow).

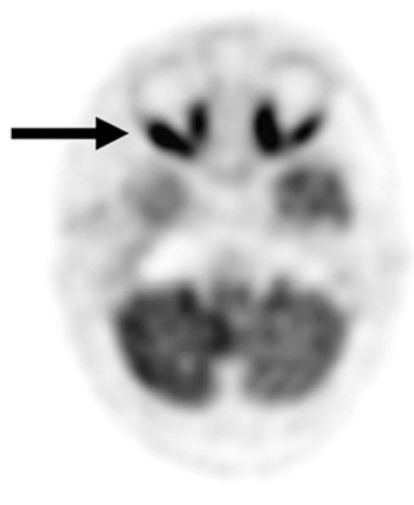


Paramount to any successful PET/CT examination is the establishment of acquisition protocols that allow highquality images to be obtained while ALARA principles are followed. In the current diagnostic imaging environment, much discussion revolves around CT dose and, more specifically, around reducing patient dose, particularly with respect to the pediatric population. Working with the technologists and radiologists in CT enables the PET/CT team to provide superior-quality imaging with a dose as minimal as possible to the patient. For patients requiring a nondiagnostic, low-dose CT scan (for attenuation correction only), we have found that using a milliamperage of 16-30 (dependant on patient size and age) has been sufficient. If a diagnostic CT scan has been requested along with the PET scan, the milliamperage is adjusted for each patient according to patient weight and the area being scanned. The CT technologists can use values established from their own protocols to ensure a minimal dose to the patient for the CT portion of the scan.

Acquisition times for the PET/CT scan range, on average, from 15 to $20 \mathrm{~min}$. Routinely, for a whole-body (skull base to mid thigh) ${ }^{18} \mathrm{~F}-\mathrm{FDG}$ scan, the PET acquisition is done at a rate of $1 \mathrm{~min} 30 \mathrm{~s}$ per bed position, with an average of $7-10$ positions per scan. The relatively short scan time becomes important in pediatric PET/CT, as many patients may be able to cooperate for short periods but may become restless or unsettled if longer imaging times are required. Restlessness can result in patient motion artifacts and poor coregistration between the PET and CT images. Even the most cooperative of patients can become uncomfortable, and motion can become a problem. Because many of the patients are having a diagnostic CT scan as part of the PET/CT scan, whole-body imaging is routinely performed with the arms up. This measure is considered dosesaving, as imaging with the arms down will require an increased CT dose to compensate for attenuation. If the patient's range of motion does not allow for having the arms up, or if the upper extremities are an area of concern, imaging will be done with the arms down. The use of sheets or pillows under the arms can alleviate some discomfort patients may experience during the scan and thereby reduce motion artifacts. Additionally, some patients may fall asleep on the scanning bed, and a cushion or head block may be useful to help prevent motion. Figures 3 and 4 demonstrate the poor coregistration and the artifacts that can be seen due to patient motion on a PET/CT scan.

\section{${ }^{18}$ F-FDG PET CARDIAC IMAGING}

Although less routine than whole-body imaging, both cardiac PET and brain PET using ${ }^{18}$ F-FDG have been demonstrated as a valuable tool in the adult population and to a lesser degree in children.

Cardiac imaging using ${ }^{18} \mathrm{~F}-\mathrm{FDG}$ plays an important role in the assessment of myocardial viability and can be particularly valuable when used in conjunction with other imaging modalities such as ${ }^{99 \mathrm{~m}} \mathrm{Tc}$-sestamibi scanning. To

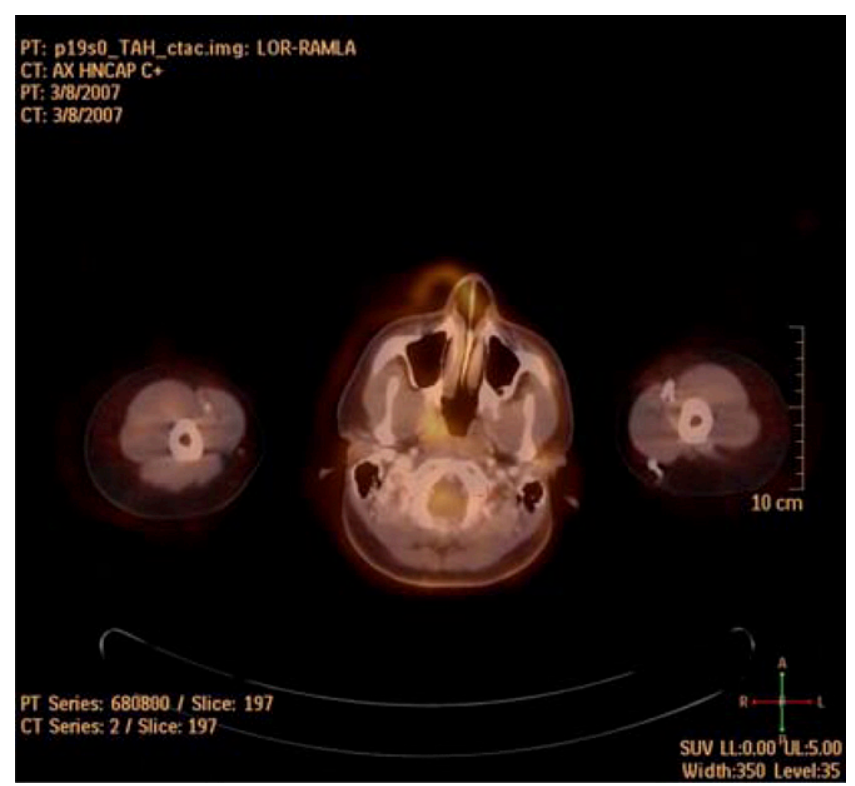

FIGURE 3. Poor registration due to motion of head between scans.

enhance ${ }^{18} \mathrm{~F}$-FDG uptake into viable myocardium, patients are administered oral or intravenous glucose before the injection of ${ }^{18}$ F-FDG. The blood glucose level has measured before intravenous glucose administration is commenced and immediately before injection of ${ }^{18} \mathrm{~F}-\mathrm{FDG}(6)$. A dose of $5-7 \mathrm{MBq}$ of ${ }^{18} \mathrm{~F}-\mathrm{FDG}$ per kilogram is injected 30 min before scanning, with intravenous glucose administration continuing throughout the uptake phase. Scanning is done at 1 bed position and with an acquisition time of 10 min. Images can be gated or nongated during the PET acquisition, based on clinical indications and the availability of software for processing gated data.

The cardiac image on the left in Figure 5 demonstrates multiple areas of both increased and decreased uptake of ${ }^{18}$ F-FDG. This patient subsequently underwent a resting sestamibi scan to further assess myocardial viability. A comparison of the 2 imaging modalities indicated hibernating myocardium in the distal anteroseptal wall, distal anterior wall, apical wall, and proximal posterolateral wall.

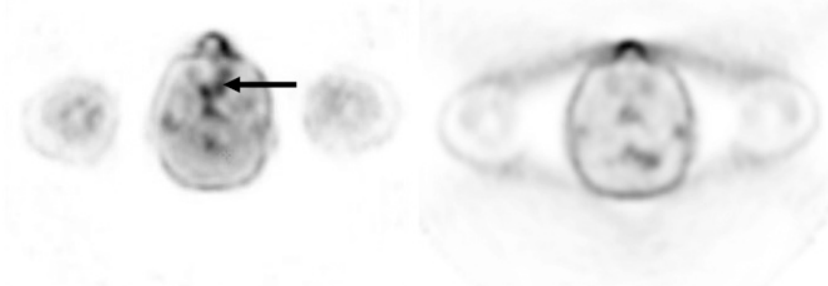

FIGURE 4. Misregistration artifact seen on attenuationcorrected image (left) but not on non-attenuation-corrected image (right). Artifact is result of patient motion between CT and PET acquisitions. 
FIGURE 5. ${ }^{18} \mathrm{~F}-\mathrm{FDG}$ cardiac uptake correlated with stress MIBI imaging.

\section{${ }^{18}$ F-FDG PET BRAIN IMAGING}

Brain tumors account for approximately $20 \%$ of all childhood cancers, with medulloblastoma the most common malignant brain tumor. Software that allows fusion or coregistration of functional PET brain images with anatomic MRI gives physicians further insight when evaluating these disease processes. ${ }^{18} \mathrm{~F}-\mathrm{FDG}$ PET is based on the similarity between ${ }^{18} \mathrm{~F}-\mathrm{FDG}$ and glucose, with ${ }^{18} \mathrm{~F}-\mathrm{FDG}$ being phosphorylated on entering the cell without being metabolized further, resulting in accumulation. This accumulation is proportional to the metabolic rate of the cell at the time of injection. ${ }^{18}$ F-FDG PET brain imaging has been studied for differentiating recurrent tumor from radiation necrosis. Areas of tumor exhibit high uptake, whereas radionecrosis typically exhibits low ${ }^{18} \mathrm{~F}$-FDG uptake (1).

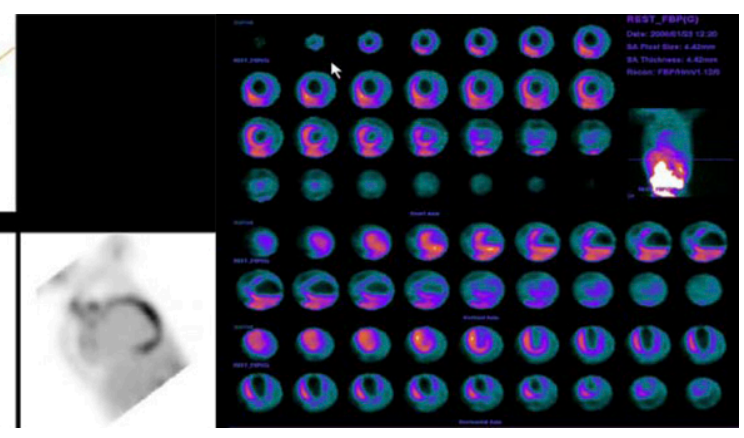

The images in Figure 6, from a ${ }^{18}$ F-FDG PET brain scan, demonstrate this principle. A discrete focus of increased ${ }^{18} \mathrm{~F}$-FDG uptake at the anterior margin of the occipital horn of the lateral ventricle was suggestive of tumor recurrence. Subsequent imaging with MRI and CT confirmed the presence of a tumor in this location.

Patient preparation for ${ }^{18} \mathrm{~F}-\mathrm{FDG}$ brains scans is similar to that for whole-body ${ }^{18} \mathrm{~F}-\mathrm{FDG}$ scanning. The patients are asked to fast for $4 \mathrm{~h}$ before injection, with the blood glucose level being measured immediately before administration of the radiotracer. The patients are asked to remain in a warm, quiet room with minimal activity for approximately 30-45 min. Scanning for the PET portion generally involves 1 bed position with a total acquisition time of $10 \mathrm{~min}$. This acquisition is performed immediately after the CT scan of
FIGURE 6. Transaxial views of MRI (top), PET (middle), and fused imaging (bottom) revealing matching defects in left hemisphere, with discrete focus of increased ${ }^{18} \mathrm{~F}-\mathrm{FDG}$ uptake in occipital horn of left lateral ventricle.
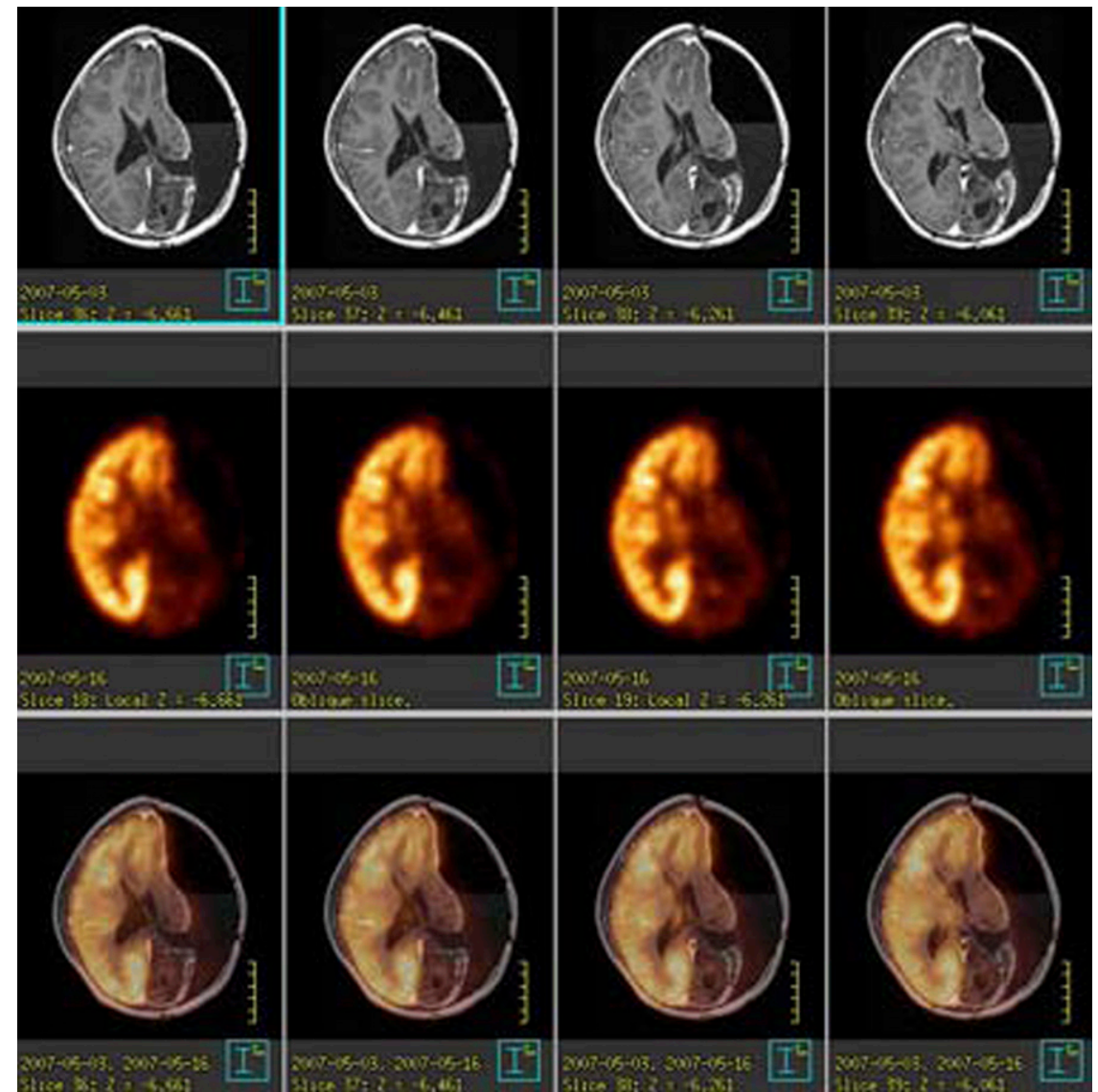
the head-a scan that will be acquired according to departmental protocol if diagnostic CT is required. If no diagnostic CT is needed, then the use of routine low-dose CT is sufficient for attenuation correction. It is essential that patients be positioned correctly for these scans and be instructed to remain motionless for the duration. The use of head holders or straps across the forehead is recommended.

\section{NORMAL VARIANTS AND DISTRIBUTION}

Brain cortex generally has an intense uptake, and the accumulation of ${ }^{18} \mathrm{~F}$-FDG in the brain is known to account for as much as $20 \%$ of total-body glucose metabolism in the fasting state (7). Uptake in the salivary glands can be variable and may be symmetric or asymmetric. Myocardial uptake can also vary significantly among patients and can range from absent to very intense uptake seen in the left and occasionally right ventricle.

Other sites of normal distribution include variable uptake in the digestive tract ( 8 ) and genitourinary system, including the collecting system, ureters, and bladder. Increased uptake may also be seen in the uterus or ovaries depending on the phase of the patient's menstrual cycle at the time of imaging. Ovarian uptake is more prominent in mid cycle (9). An example is seen in Figure 7. This patient demonstrated an uptake pattern consistent with an obstructed kidney and ureter (due to a mass) but also showed ovarian uptake.

Muscle uptake can vary in relation to vigorous exercise in the days preceding the scan but may also be enhanced by stress-induced muscle tension, hyperventilation, crying, or coughing (as seen by uptake in the diaphragm). Figure 8 shows a patient with posttransplantation lymphoproliferative disease who was crying for much of the uptake phase.
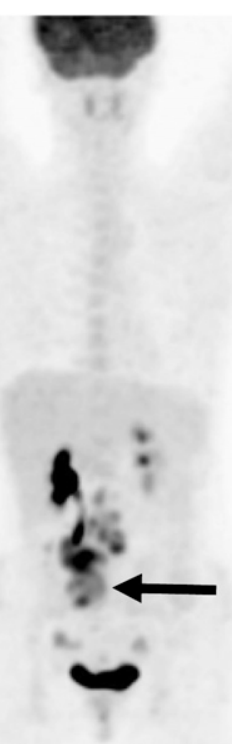

FIGURE 7. Uptake in obstructed kidney, ureter, ovaries, and primary neoplastic lesion (arrow).

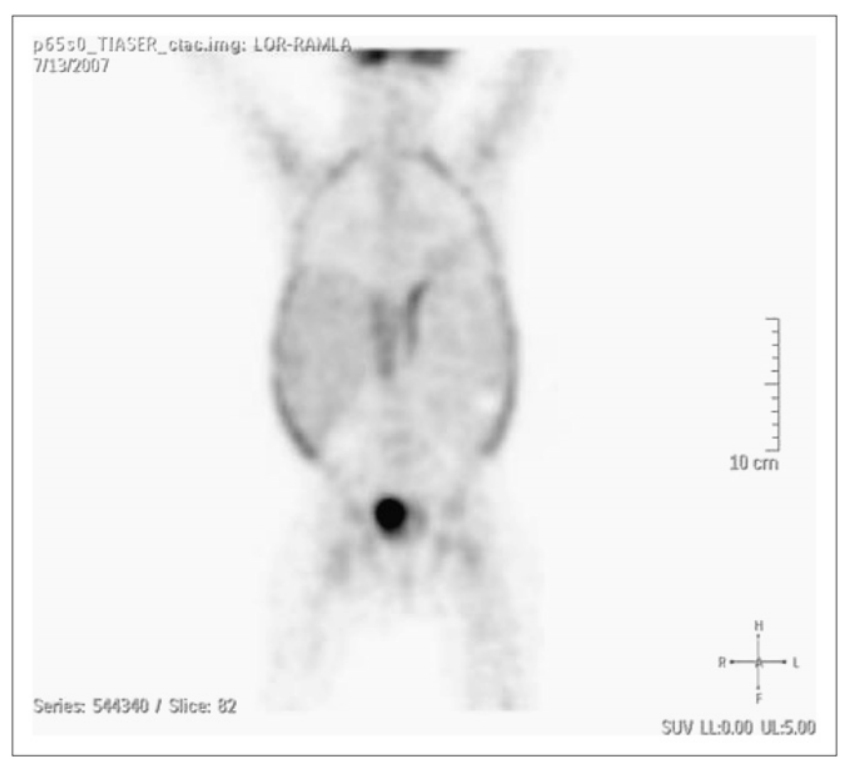

FIGURE 8. Image of $2-y$-old child who was crying during uptake phase.

Uptake in the diaphragm, abdominal wall, and intercostal muscles is noted.

Although muscle uptake tends to be symmetric, with mild to moderate linear activity (7), it can be focal or unilateral as demonstrated in a patient (Fig. 9) who fell asleep for the uptake phase with his head angled toward the left. Unilateral uptake in the left neck muscle was noted.

Thymus uptake is a normal variant in young adults and children that can be characterized by uptake in the shape of an upside-down $\mathrm{V}$ around the mediastinum. Bone marrow may or may not appear on whole-body images but usually presents as faint activity (less than liver uptake) and most often is seen in the vertebral bodies. Although focal activity within bone marrow is always suggestive of an abnormality, uniformly diffuse increased bone marrow activity can be

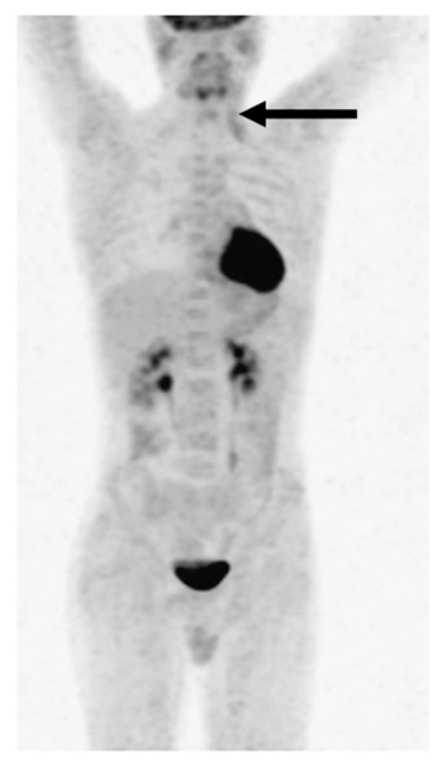

FIGURE 9. Asymmetric muscle uptake due to head position during uptake phase. 


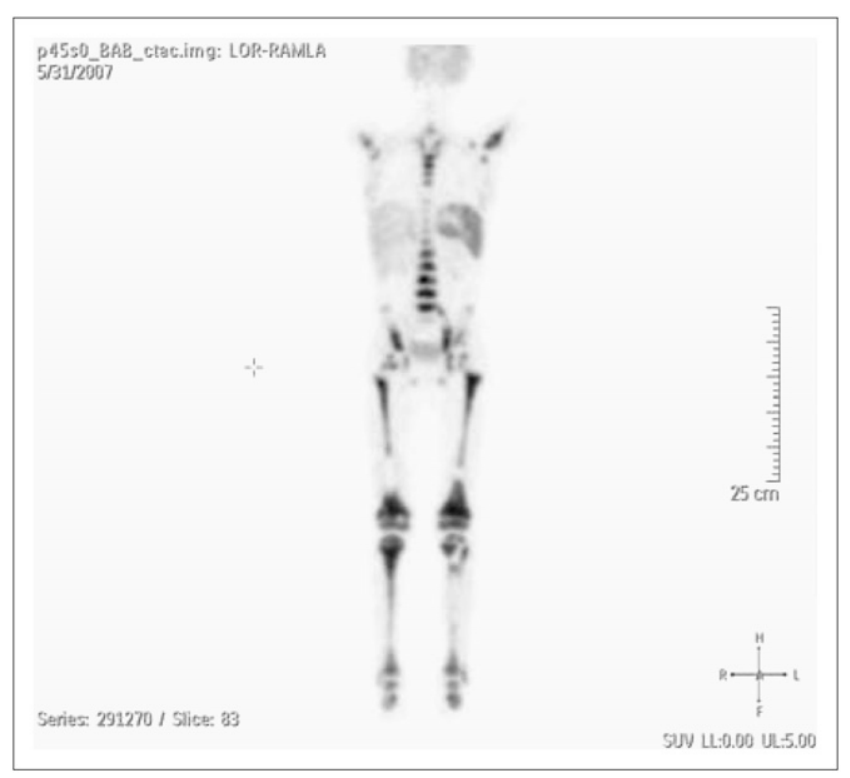

FIGURE 10. Diffuse bone marrow uptake in patient who was receiving granulocyte colony-stimulating factor.

seen in patients who have recently received chemotherapy or are taking granulocyte colony-stimulating factor (7), as demonstrated in Figure 10. It is for this reason that it is imperative to obtain a full and adequate patient history before injection and to have this information readily available to the physicians who will be reading the scans. It is also important to note that increased uptake can be seen in the growth plates of many pediatric patients and is considered a normal pattern of distribution.

\section{BROWN FAT}

Symmetric uptake in brown adipose tissue in the supraclavicular, midaxillary line, and paraspinal regions can mimic pathologic uptake (Fig. 11) (8). In our experience at The Hospital for Sick Children, up to $20 \%$ of children exhibit

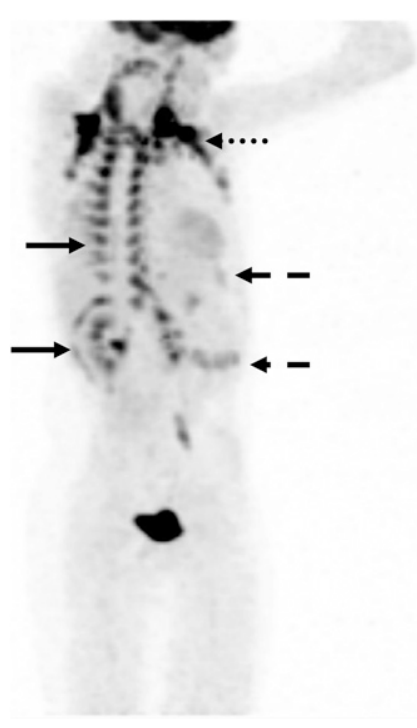

FIGURE 11. Brown fat uptake in costal vertebral junction and around kidney (solid arrow), clavicle (dotted arrow), abdominal wall, and intercostal region (dashed arrow). brown adipose uptake on PET/CT scans (10). It can be distinguished by intense ${ }^{18} \mathrm{~F}$-FDG uptake seen on high-quality PET scans, with no corresponding abnormality visualized on CT (8). Maintaining a warm and calm environment can help reduce brown adipose uptake in most patients; however, for some patients predisposed to such uptake, the use of $\beta$ blockers or pharmaceuticals such as diazepam may become necessary. This need can be reviewed before arrival of the patient so that appropriate preparations can be made. In colder climates, when possible it can be beneficial to allow patients a period of time to warm up and relax when coming in from outdoors to reduce brown adipose uptake.

\section{CONCLUSION}

The use of PET/CT in children is quickly growing in importance. The combination of high-quality PET scans with diagnostic or low-dose CT scans aids physicians in the staging, therapy planning, and treatment of many pediatric oncology patients. Fusion of the physiologic PET scan with the anatomic CT scan can help in distinguishing disease from other physiologic processes and can be an invaluable tool for referring physicians when they are evaluating for recurrence of disease. This relatively noninvasive scan can provide, through a single study, information and insight that in previous years required multiple scans using several different imaging modalities. Although still young in its development, pediatric PET/CT shows a promising future. As new technology becomes available, scanners will continue to evolve and PET/CT will continue to play a key role in pediatric imaging. Through extensive research and clinical applications, we will continue to unravel some of the mysteries surrounding pediatric disease processes and will work toward finding ways to help in the treatment and cure of many of these illnesses.

\section{REFERENCES}

1. Fisher M, Phillips C, Charron M, eds. Practical Pediatric PET Imaging. New York, NY: Springer; 2006:3-20,175-209.

2. Hamblen SM, Lowe VJ. Clinical ${ }^{18} \mathrm{~F}-\mathrm{FDG}$ oncology patient preparation techniques. J Nucl Med Technol. 2003;31:3-7.

3. Kaste SC. Issues specific to implementing PET-CT for pediatric oncology: what have we learned along the way. Pediatr Radiol. 2004;34:205-213.

4. Veitch TA. Pediatric nuclear medicine, part I: developmental cues. J Nucl Med Technol. 2000;28:3-7.

5. Hossein J, Connolly L, Shulkin B. PET imaging in pediatric disorders. In: Valk P, Bailey D, Townsend D, et al., eds. Positron Emission Tomography: Basic Science and Clinical Practice. London, U.K.: Springer-Verlag; 2003:756.

6. Rickers C, Sasse K, Buchert R, et al. Myocardial viability assessed by positron emission tomography in infants and children after the arterial switch operation and suspected infarction. J Am Coll Cardiol. 2000;36:1676-1683.

7. Abouzied MM, Crawford ES, Nabi HA. ${ }^{18}$ F-FDG imaging: pitfalls and artifacts. J Nucl Med Technol. 2005;33:145-155.

8. Kostakoglu L, Mirtcheva R, Goldsmith SF. PET-CT fusion imaging in differentiating physiologic from pathologic FDG uptake. Radiographics. 2004; 24:1411-1431.

9. Ahu Z, Wang B, Cheng W, et al. Endometrial and ovarian F-18 FDG uptake in serial PET studies and the value of delayed imaging for differentiation. Clin Nucl Med. 2006;31:781-787.

10. Shammas A, Lim R, Charron M. Brown adipose tissue in pediatric FDG PET/CT imaging. Educational exhibit presented at: RSNA 2007: Connecting Radiology; November 25-30; Chicago, IL. Code LL-NM2734. 